\title{
Facile method for spectroscopic examination of radical ions of hydrophilic carotenoids
}

\author{
K. Razi Naqvi, ${ }^{* a}$ Thor Bernt Melø, ${ }^{a}$ Tamás Jávorfi, ${ }^{b}$ Sergio González-Pérez ${ }^{c}$ \\ and Juan B. Arellano ${ }^{a c}$
}

Received 18th March 2009, Accepted 7th May 2009

First published as an Advance Article on the web 9th June 2009

DOI: $10.1039 / b 905454 \mathrm{e}$

\begin{abstract}
Hydrophilic carotenoids, unusual members of an intrinsically hydrophobic family, and their radical ions are important reactants. An all-optical method for generating singly charged radical ions of a hydrophilic carotenoid (Car) is described. It relies on photolyzing an aqueous mixture of Car and a photoionizable auxiliary solute $(\mathbf{A})$, and making conditions conducive to the capture, by Car, of the hydrated electron $\left(\mathrm{e}_{\mathrm{aq}}^{-}\right.$) or the positive hole in $\mathbf{A}^{\bullet^{+}}$or both. When $\mathbf{A}$ is Trolox ( $\mathrm{TOH}$ ), only $\mathrm{e}_{\mathrm{aq}}^{-}$can be captured, since $\mathrm{TOH}^{\bullet+}$ deprotonates too rapidly to be a hole donor; when $\mathbf{A}$ is Trolox methyl ether (TOMe), both $\mathrm{Car}^{\bullet-}$ and $\mathrm{Car}^{\bullet+}$ are formed, since $\mathrm{TOMe}^{\bullet+}$ lives long enough to transfer its positive hole to Car; formation of $\mathrm{Car}^{\bullet-}$ is prevented under aerobic conditions.
\end{abstract}

\section{Introduction}

Carotenoids form a large group of brightly colored molecules, noted for their manifold beneficial roles in the plant and animal kingdoms; ${ }^{1-3}$ humans set great store by a carotenoid-rich diet because it is believed to lower the risk of cancer and cardiovascular disease. As a general rule, carotenoids are hydrophobic, but a few exceptions, among which $\operatorname{crocin}^{4}$ and norbixin ${ }^{5}$ are the best known, do exist, and hold much promise for parenteral applications. ${ }^{6-10}$ Our purpose here is to fill a gap in the investigation of mono-positive and mononegative carotenoid radical ions, which are considered to be important reaction intermediates.

The most successful strategy for spectroscopic investigations of singly charged radical ions of carotenoids relies on the production, through pulse radiolysis, of solvated electrons and solvent-derived radical cations, ${ }^{1-13}$ the latter may be viewed, in the present context, as carriers of positive holes. Carotenoid (Car) molecules subsequently capture the solvated electrons as well as the positive holes, and the resulting radical ions, which have strongly allowed absorption transitions in the visible and near infrared regions, can be easily detected with the help of kinetic spectroscopy. In non-protic solvents, both $\mathrm{Car}^{\bullet-}$ and $\mathrm{Car}^{\bullet}{ }^{+}$are formed, but the formation of $\mathrm{Car}^{\bullet-}$ can be easily prevented by saturating the solution with a gaseous electron scavenger, such as $\mathrm{N}_{2} \mathrm{O}$ or $\mathrm{O}_{2} \cdot{ }^{11,14}$ In alcohols ${ }^{11}$ or alcohol-water mixtures, ${ }^{12}$ where the positive hole does not survive for long, only $\mathrm{Car}^{\bullet-}$ is observed. ${ }^{11}$ It is possible to form $\mathrm{Car}^{\bullet+}$ in polar solvents, ${ }^{15}$ including water, ${ }^{16}$ by using the triplet state of 1-nitronaphthalene

\footnotetext{
${ }^{a}$ Department of Physics, Norwegian University of Science and Technology (NTNU), N-7491, Trondheim, Norway.

E-mail: razi.naqvi@ntnu.no; Fax: +47 73597710;

Tel: + 4773591853

${ }^{b}$ Diamond Light Source Ltd, Didcot, Oxfordshire, United Kingdom OX11 ODE

${ }^{c}$ Instituto de Recursos Naturales y Agrobiología de Salamanca

(IRNASA-CSIC), Apdo. 257, 37071 Salamanca, Spain
}

as an electron acceptor. A strategy for producing both $\mathrm{Car}^{\bullet}{ }^{+}$and $\mathrm{Car}^{\bullet-}$ in protic solvents, using a single pump-probe technique, is still lacking.

The dearth of spectroscopic information concerning singly charged radical ions of hydrophilic carotenoids, and the recent growth of interest in using water-soluble carotenoids as pharmaceutical or nutraceutical antioxidants, ${ }^{17,18}$ prompted us to explore the possibility of generating these species via photoionization of an auxiliary solute. A suitable auxiliary solute (A) for this purpose would have the following traits: commercial availability, hydrophilicity, and an appreciable yield of photoionization; a negligible yield for triplet formation is highly desirable, for that would forestall the sensitization of Car triplets, which are known to have intense triplet-triplet absorption bands; $\mathbf{A}^{\bullet+}$ should be long-lived, so that it may serve as a hole donor (electron acceptor); it should be possible to generate only $\mathrm{Car}^{\bullet+}$ or $\mathrm{Car}^{\bullet-}$ by selectively removing, through a fast chemical reaction, either the hydrated electron $\left(\mathrm{e}_{\mathrm{aq}}^{-}\right)$or $\mathbf{A}^{\boldsymbol{\bullet}^{+}}$, but the removal should not complicate the analysis of transient difference spectra. We have found that Trolox (TOH), a hydrophilic vitamin E derivative, and Trolox methyl ether (TOMe), considered jointly, meet all of the above requirements; each fails on only a single count, but where one falls short the other does not. To be specific: $\mathrm{TOMe}^{\bullet+}$ is long-lived, but there seems to be no convenient way of disabling it as a hole donor; fortunately, $\mathrm{TOH}^{\bullet}+$ is too unstable to serve as a hole donor, because it deprotonates rapidly to form $\mathrm{TO}^{\bullet}$, a relatively inert radical with a well-known absorption spectrum. ${ }^{19,20}$ What pulse radiolysis permits in non-protic solvents - namely observation of both $\mathrm{Car}^{\bullet-}$ and $\mathrm{Car}^{\bullet+}$, or of only $\mathrm{Car}^{\bullet+}$ - can also be accomplished in aqueous solutions, with equal if not greater ease, by means of flash photolysis. We will illustrate the foregoing remarks by presenting some time-resolved spectra recorded with the aid of a multi-channel pump-probe spectrometer with $\mu$ s time resolution. ${ }^{21}$ 


\section{Results and discussion}

Since Bors et al. $^{22}$ have already used pulse radiolysis for recording the spectra of the radical anions of crocin and crocetin $(\mathbf{C})$ we chose these molecules for testing our method; here we will confine ourselves to the spectra obtained by using C, whose structure is shown in Scheme 1. As we will be making frequent reference to the difference spectra of various photoproducts, it will be convenient to label the spectrum of a species as $S_{p}$, with a different subscript for each photoproduct: $\mathrm{e}$ for $\mathrm{e}_{\mathrm{aq}}^{-}, \mathrm{n}$ for the neutral radical $\mathrm{TO}^{\bullet}, \mathrm{d}$ for the hole donor $\mathrm{TOH}^{\bullet+}$, a for the radical anion $\mathbf{C}^{\bullet-}$ and $\mathrm{c}$ for the radical cation $\mathbf{C}^{\bullet}+$. The change in absorbance recorded at a delay of $d \mu$ s will be denoted by $\Delta @ d$.

Since spectrophotometric data on TOMe do not seem to be available, we undertook a comparison of the absorption spectra of $\mathrm{TOH}$ and TOMe at $\mathrm{pH} 8.9$, which is high enough to ensure ionization of the carboxylic group in each compound but not so high as to permit ionization of the hydroxyl group of TOH. The spectra of the two auxiliary solutes, shown in Fig. 1a, bear great similarity to the absorption spectra of $\alpha$-tocopherol (EOH) and $\alpha$-tocopherol methyl ether in methanol; ${ }^{23}$ in both cases, methylation brings about a blue-shift and lowers the peak value of $\varepsilon$, the molar absorption coefficient. Plots showing the $\mathrm{pH}$-dependence of the absorption spectrum of TOH $(7.4 \leq \mathrm{pH} \leq 12.9)$ are shown in Fig. 1b; the spectrum of TOMe does not change with $\mathrm{pH}$.

Flash-induced difference spectra of argon-bubbled aqueous solutions of $\mathrm{TOH}(\mathrm{pH} 11.8)$ at delays of 1 and $50 \mu$ s are displayed in Fig. 1c. On the basis of previous studies of TOH and related sytems, ${ }^{19,20,24} \Delta @ 1$ can be recognized as a superposition of $\mathrm{S}_{\mathrm{e}}$, which is known to have a broad structureless absorption band peaking at about $710 \mathrm{~nm}$, and $S_{n}$ (with peaks around 315 and $435 \mathrm{~nm}$ ). At delays longer than about $30 \mu \mathrm{s}$ in argon-saturated solutions, only $\mathrm{S}_{\mathrm{n}}$ was present; this was also the case at shorter delays in solutions saturated with $\mathrm{O}_{2}$ or $\mathrm{N}_{2} \mathrm{O}$, which are well known scavengers of $\mathrm{e}_{\mathrm{aq}}^{-} \cdot{ }^{11,14}$ In the spectral region where absorption by $\mathrm{TOH}$ is negligible, the shapes of the spectra $S_{n}$ and $S_{e}$ did not change with $\mathrm{pH}$. However, the yields of the two photoproducts were found to be larger under more alkaline conditions; since the yield of $\mathrm{e}_{\mathrm{aq}}^{-}$ determines the amplitude of the signal contributed by $\mathbf{C}^{\bullet-}$, and since the shapes of $S_{a}$ and $S_{c}$ were also found to be insensitive to $\mathrm{pH}$, all the transient spectra reported here were recorded at $\mathrm{pH} 11.9$.

The corresponding spectra obtained by using TOMe instead of TOH are plotted in Fig. 1d. In this case $\Delta @ 50$ (with peaks located at approximately 314 and $490 \mathrm{~nm}$ ) can be identified with $S_{d}$ on account of its similarity to the spectrum of the radical cation of an analogue of $\mathrm{EOH}$ in which the phenolic $\mathrm{H}$-atom is lacking; this cation, generated electrochemically in acetonitrile, displayed peaks at 313 and $482 \mathrm{~nm} \cdot{ }^{25}$ Our<smiles>CC(/C=C/C=C(\C)C(=O)O)=C\C=C\C=C(C)\C=C\C=C(/C)C(=O)O</smiles>

Scheme 1 Chemical structure of crocetin.

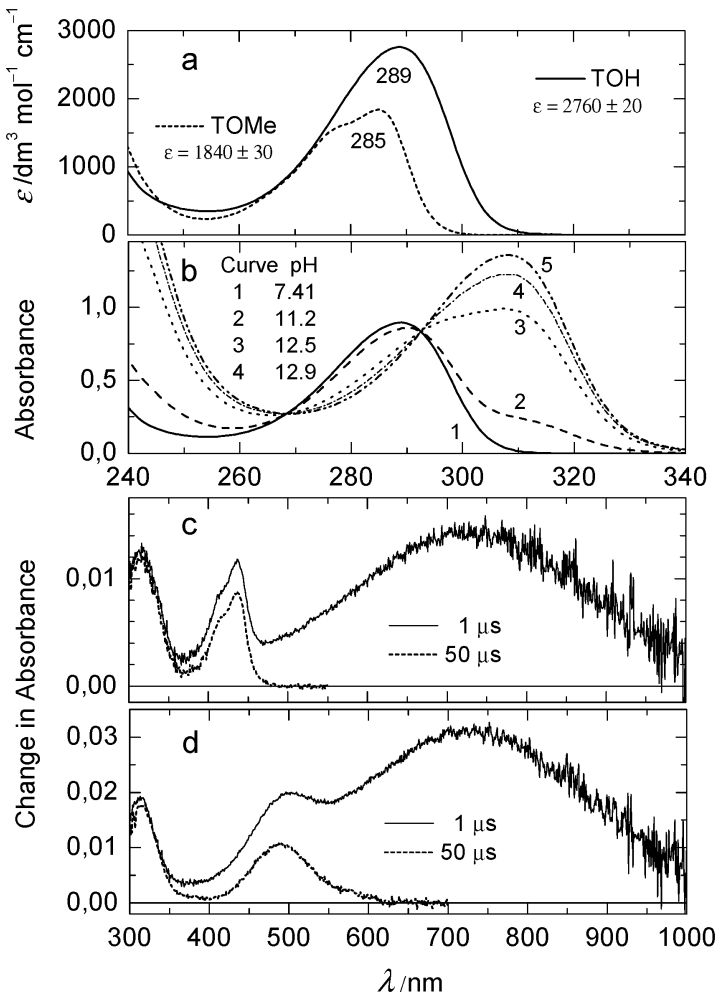

Fig. 1 Absorption spectra of the auxiliary solutes and their long-lived transient photoproducts in aqueous solutions. (a) Plots of molar absorption coefficients $\varepsilon$ (at $\mathrm{pH}$ 8.9); the peak value is indicated and the corresponding wavelength is given near the top of the spectrum. (b) Plots showing the $\mathrm{pH}$-dependence of the absorption spectrum of Trolox in aqueous solutions. (c) and (d) Transient absorption difference spectra of Ar-bubbled aqueous solutions (pH 11.8) of Trolox (ca. $0.3 \mathrm{mM})$ and Trolox methyl ether $(c a .0 .5 \mathrm{mM})$ at two delays.

assignment is also consistent with the crucial result, to be presented below, that $\mathrm{TOMe}^{\bullet+}$ is able to donate its hole to $\mathbf{C}$. On this basis $\Delta @ 1$ can be immediately interpreted as the superposition of $\mathrm{S}_{\mathrm{e}}$ and $\mathrm{S}_{\mathrm{d}}$; in $\mathrm{N}_{2} \mathrm{O}$-saturated solutions, only $\mathrm{S}_{\mathrm{d}}$ is observed.

The results summarized in Fig. 1c and d show that, regardless of whether $\mathbf{A}=\mathrm{TOH}$ or $\mathbf{A}=\mathrm{TOMe}$, photoexcitation leads to the formation of $\mathrm{e}_{\mathrm{aq}}^{-}$and the solute radical cation $\mathbf{A}^{\bullet+}$; that the cation $\mathrm{TOH}^{\bullet}$ deprotonates to form $\mathrm{TO}^{\bullet}$ on the sub-ns time scale is already known, ${ }^{26}$ but the occurrence of electron photoejection from TOMe and the long lifetime of $\mathrm{TOMe}^{\bullet+}$ has not been reported previously (to the best of our knowledge).

We turn now to the photo-reactions of aqueous solutions of $\mathbf{C}$ (in the absence of $\mathbf{A}$ ). Craw and Lambert, ${ }^{27}$ who used a frequency-doubled ruby laser $(347 \mathrm{~nm})$ as their pump source, reported that the laser-induced change in absorbance was less than 0.001 , and concluded that the quantum yield of intersystem crossing was less than $1 \%$. When we interposed a long-pass filter (cutoff wavelength $340 \mathrm{~nm}$ ) between the pump flash and the sample, the resulting difference spectrum could not be distinguished from the noisy baseline in our experiments. However, when $\mathbf{C}$ was photolyzed by the same unfiltered pump light as that used for exciting $\mathrm{TOH}$ and TOMe, we observed a difference spectrum that could be 
reconstructed, on the basis of the results presented below, as a superposition of $\mathrm{S}_{\mathrm{a}}$ and $\mathrm{S}_{\mathrm{c}}$; in aerated solutions, only $\mathrm{S}_{\mathrm{c}}$ was present (with a peak absorbance around 0.01). Using a ns laser photolysis apparatus (see below), we have verified that $S_{e}$ and $S_{c}$ are present immediately after the laser pulse, and the former is replaced within a few $\mu$ s by $S_{a}$ (data not shown). Although photolysis by pulsed laser light of $266 \mathrm{~nm}$ (where Car absorbance is usually rather low) does provide an alternative method for recording the spectra of singly charged radical ions of a hydrophilic Car, it cannot compete, in terms of signal-to-noise ratio (SNR), with the method advocated here, where the Car concentration is kept low (around $10 \mu \mathrm{M}$ ) in order to permit measurements even in the region where Car absorbs strongly.

Unlike $\mathbf{C}^{\bullet-}$, which can be generated only through electron capture, $\mathbf{C}^{\bullet+}$ can be produced in two ways: photoionization (as mentioned above) and hole capture (as demonstrated below). It seems worthwhile to make a distinction between a 'prompt' radical cation produced directly by photoionization and a 'delayed' radical cation formed by the capture of a positive hole. It is now easy to foresee the outcome of experiments involving photolysis of an aqueous mixture of $A$ and $\mathbf{C}$. Since we are no longer interested in $S_{e}$ and $S_{d}$, we will consider the situations where the delay is sufficiently long to permit complete capture (by $\mathbf{C}$ in an Ar-bubbled solution) of $\mathrm{e}_{\mathrm{aq}}^{-}$and the positive hole of $\mathrm{TOMe}^{\bullet+}$; under these conditions, the difference spectrum will be a superposition of $S_{a}$ and $S_{c}$, if $\mathbf{A}=$ TOMe, or of $S_{a}, S_{n}$ and $S_{c}$ (contributed solely by prompt $\mathbf{C}^{\bullet+}$ ), if $\mathbf{A}=\mathrm{TOH}$. Since $\mathrm{S}_{\mathrm{a}}$ can be eliminated simply by aerating the sample, the task of obtaining only $\mathrm{S}_{\mathrm{c}}$ presents no problems.

Once $S_{c}$ becomes available, $S_{a}$ can be obtained in at least two ways. One can subtract $S_{c}$, obtained by photolyzing an aerated TOMe-C mixture, from the difference spectrum of an Ar-bubbled mixture with the same composition. The subtraction would be trustworthy only if variations in the output of the pump flash were negligible, which is not always true. A much better alternative is to subtract $S_{n}$ and $S_{c}$ (prompt) from the difference spectrum of an Ar-bubbled TOH-C mixture; the influence of the shot-to-shot variation in the pump output (up to $10 \%$ in our experiments) becomes negligible in this case because the peak amplitudes of $S_{n}$ and $S_{c}$ are less than one tenth of the maximum value of $S_{a}$. It is imperative, of course, to record each unwanted spectrum with a good SNR; the noise content of $S_{n}$ was reduced by signal averaging followed by setting the absorbance to zero for wavelengths longer than $500 \mathrm{~nm}$, and the quality of $\mathrm{S}_{\mathrm{c}}$ (prompt) was improved by replacing it with a suitably scaled version of $S_{c}$ shown below (Fig. 2), which is in fact a sum of $S_{c}$ (prompt) and $S_{c}$ (delayed).

Only one other point needs elaboration before we present the difference spectra $\mathrm{S}_{\mathrm{c}}$ and $\mathrm{S}_{\mathrm{a}}$, which are plots of $\Delta A^{ \pm}(\lambda)$ against $\lambda$, where $A$ represents absorbance and the superscript (plus or minus) has been added to specify the charge of the radical ion. One can write $\Delta A^{ \pm}=A_{\text {inc }}-A_{\text {dec }}$, where $A_{\text {inc }}$ and $A_{\text {dec }}$ represent, respectively, the increment in absorbance due to the formation of $\mathbf{C}^{\bullet \pm}$ and the consequent decrement due to the loss of $\mathbf{C}$ by the same amount. That is to say, $\Delta A^{ \pm}=$ $\ell\left(\varepsilon^{ \pm}-\varepsilon^{0}\right)\left[\mathbf{C}^{\bullet \pm}\right]$, where $\ell$ is the optical path length and $\varepsilon^{ \pm}$and

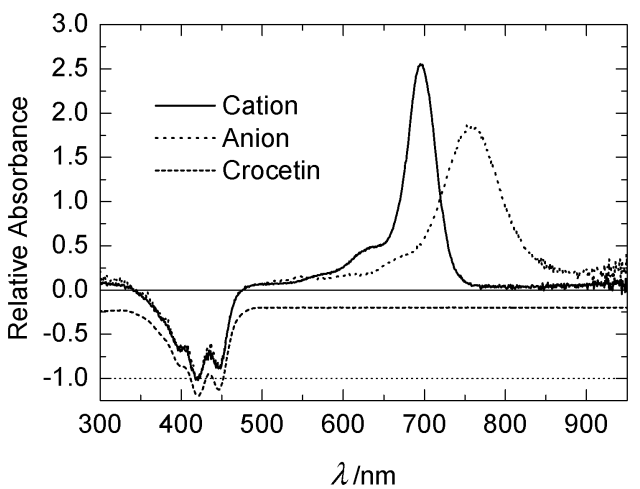

Fig. 2 Transient absorption difference spectra of singly charged radical ions of crocetin in aqueous solutions $(\mathrm{pH}$ 11.8). The spectra have been normalized by dividing the absorbance values by the magnitude of the peak negative absorbance. The absorption spectrum of crocetin (normalized to have a peak value of -1 , and displaced downward for the sake of clarity) is also plotted. The spectra of crocetin and its radical ions were found to be insensitive to $\mathrm{pH}$ over the range $8.5-13$.

$\varepsilon^{0}$ denote the molar absorption coefficients of $\mathbf{C}^{\bullet \pm}$, and $\mathbf{C}$, respectively. Since, in the region where the signals take large negative values, the shapes of $S_{c}$ and $S_{a}$ are very similar to the shape of the absorption spectrum of $\mathbf{C}$, a quantitative expression can be given to the difference spectra by plotting the ratios $R^{ \pm} \equiv \Delta A^{ \pm} /\left|\Delta A_{\min }^{ \pm}\right|$, where $\Delta A_{\min }^{ \pm}$is the minimum value of $\Delta A^{ \pm}$. In a spectral region where the only absorbing species is a radical ion, the relative absorbance $R^{ \pm}$can be identified with the ratio $\varepsilon^{ \pm} / \varepsilon_{\max }^{0}$, where $\varepsilon_{\max }^{0}$ is the peak value of the molar absorption coefficient of $\mathbf{C}$.

Plots of $R^{ \pm}$are displayed in Fig. 2; the absorption spectrum of $\mathbf{C}$ has also been plotted (after multiplication with a negative constant) and with a vertical offset, without which it would become indistinguishable from the bleaching part of $S_{c}$ and $S_{a}$. Our spectra of the radical ions (of both crocin and $\mathbf{C}$ ) confirm the results reported by Bors et al. ${ }^{22}$ The two published values of $\varepsilon_{\max }^{0}$ for $\mathbf{C}$ agree within $10 \%, 28,29$ if we accept the more recent determination, and set $\varepsilon_{\max }^{0}=1.0 \times$ $10^{5} \mathrm{dm}^{3} \mathrm{~mol}^{-1} \mathrm{~cm}^{-1}$, we get $\varepsilon_{\max }^{0}=2.6 \times 10^{5} \mathrm{dm}^{3} \mathrm{~mol}^{-1} \mathrm{~cm}^{-1}$ (with $\lambda_{\max }^{+}=695 \mathrm{~nm}$ ) and $\varepsilon_{\max }^{-}=1.9 \times 10^{5} \mathrm{dm}^{3} \mathrm{~mol}^{-1} \mathrm{~cm}^{-1}$ (with $\lambda_{\max }^{-}=759 \mathrm{~nm}$ ). As has been found to be the case with other carotenoids, the values of $\varepsilon_{\max }^{ \pm}$are appreciably larger than the corresponding value of $\varepsilon_{\max }^{0}$. A more interesting feature is the red-shift of $S_{a}$ with respect to $S_{c}$, which was also found to be the case for crocin (data not shown); apart from crocin and $\mathbf{C}$, the only other example of this ordering is canthaxanthin, ${ }^{11}$ also a symmetric molecule with a conjugated carbonyl group at each end.

As a final check on the reliability of $S_{a}$ and $S_{c}$, we will now show, with the aid of Fig. 3, that a set of transient spectra recorded at different delays after photolyzing an Ar-bubbled TOMe-C mixture can be reproduced, with the help of the spectrum reconstruction method, ${ }^{30}$ as linear combinations of $S_{a}$ and $S_{c}$, showing that each spectrum represents a mixture of $\mathbf{C}^{\bullet+}$ and $\mathbf{C}^{\bullet-}$ in different proportions. The reconstruction, near-perfect for longer delays (with $R^{2}>0.998$ ), is illustrated in Fig. 3, where the reconstruction of $\Delta @ 50$ is presented. 


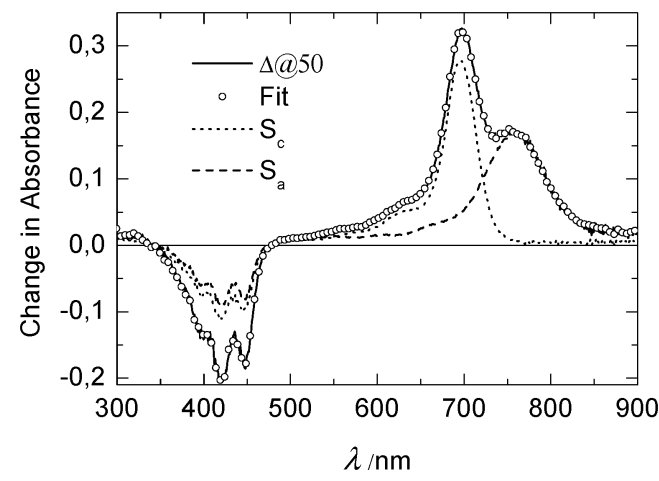

Fig. 3 Transient difference spectrum recorded $50 \mu$ s after photolyzing an Ar-bubbled aqueous mixture (pH 11.8) of crocetin $(c a .10 \mu \mathrm{M})$ and Trolox methyl ether $(c a .0 .5 \mathrm{mM})$. The spectrum can be successfully reproduced as a superposition of the spectrum of the radical cation $\left(\mathrm{S}_{\mathrm{c}}\right)$ and of the radical anion $\left(\mathrm{S}_{\mathrm{a}}\right)$; the component spectra and the superposition (labelled Fit) are also plotted.

The results of the reconstructions can be summarized by the following list of $\left(d, R^{2}\right)$ : $(50,0.9994),(25,0.9992),(15,0.9989)$, $(5,0.9985),(1,0.9958)$. The deterioration of the fit with decreasing $d$ is a reflection of the fact that $S_{d}$, though very small, is not negligible at shorter delays.

\section{Experimental}

Trolox (TOH) and Trolox methyl ether (TOMe) were purchased from Sigma-Aldrich Co. (St. Louis, MO); purified samples of the carotenoids crocin and crocetin $(\mathbf{C})$, donated by Dr Vassilia Partali, were used within a few hours of purification. The solutes were dissolved in ultrapure Milli-Q water, and the $\mathrm{pH}$ of the solution was adjusted (between 8.5 and 13) by adding the requisite amount of concentrated $\mathrm{NaOH}$. Though TOMe is only sparingly soluble in water, it was possible to prepare solutions with $\hat{A}_{1} \simeq 1$, where $\hat{A}_{1}$ denotes the peak absorbance for a path length of $1 \mathrm{~cm}$; in the case of $\mathbf{C}$, solutions with a monomeric absorption spectrum $^{29}$ and $\hat{A}_{1} \simeq 1$ could be obtained under alkaline conditions ( $\mathrm{pH} \geq 8$ ).

The transient absorption spectra were acquired by using a multichannel pump-probe spectrometer with $\mu$ s time resolution and a wide spectral range $(200-1000 \mathrm{~nm})$ that has been described elsewhere. ${ }^{21,31} \mathrm{~A}$ xenon flash (of about $5 \mu$ s duration) is used as the pump source, and the resulting change in absorbance is recorded, using a right-angle arrangement, with the aid of a probing flash (of about $2 \mu$ s duration) delayed with respect to the pump. Some spectra (covering a $300 \mathrm{~nm}$ wide spectral window) were also recorded by using a ns laser photolysis instrument whose major components have been described earlier; ${ }^{32}$ the main purpose of these measurements was to complement the flash photolysis data by confirming that excitation of crocin and crocetin by a $266 \mathrm{~nm}$ pulse also leads to photoionization. In all experiments, the concentrations of $\mathrm{TOH}$, TOMe and $\mathbf{C}$ were close to $0.3 \mathrm{mM}, 0.5 \mathrm{mM}$ and $10 \mu \mathrm{M}$, respectively. For expelling air, the solution to be examined was bubbled with $\mathrm{Ar}$ or $\mathrm{N}_{2} \mathrm{O}$ for at least 30 minutes prior to taking a measurement. All measurements were made at room temperature $\left(23 \pm 1{ }^{\circ} \mathrm{C}\right)$.

\section{Conclusion}

The work described above may be seen as a sequel to an earlier investigation $^{31}$ which revealed that photoexcitation of solutions of $\mathrm{EOH}$ in acetonitrile leads to the formation of $\mathrm{EO}^{\bullet}$ and two other products, $\mathrm{EOH}^{\bullet+}$ and triplet $\mathrm{EOH}$, each of which is sufficiently long-lived to be able to donate its hole and electronic excitation (respectively) to a Car. It is remarkable that the triplet yields of TOH and TOMe in aqueous solutions turned out to be negligible, making them ideal auxiliary solutes for our purpose. Further work is needed to find out what modifications, if any, might extend the applicability of our strategy to hydrophobic carotenoids.

\section{Acknowledgements}

This work was funded by the Research Council of Norway (Project No. 191102) and the Spanish Ministry of Science and Education (Grant No. BFU2007-68107-C02-02/BMC).

\section{References}

1 S. A. Goff and H. J. Klee, Science, 2006, 311, 815-819.

2 B. Demmig-Adams and W. W. Adams, Science, 2002, 298, 2149-2153.

3 F. Böhm, J. H. Tinkler and T. G. Truscott, Nat. Med., 1995, 1, 98-99.

4 M. van Calsteren, M. C. Bissonnette, F. Cormier, C. Dufresne, T. Ichi, J. C. Y. LeBlanc, D. Perreault and I. Roewer, J. Agric. Food Chem., 1997, 45, 1055-1061.

5 F. Bouvier, O. Dogbo and B. Camara, Science, 2003, 300, 2089-2091.

6 K. Abe, M. Sugiura, Y. Shoyama and H. Saito, Brain Res., 1998, 787, 132-138.

7 J. L. Gainer, US pat. 2008/0255246, 2008.

8 L. J. Giassi, M. J. Gilchrist, M. C. Graham and J. L. Gainer, J. Trauma, 2001, 51, 932-938.

9 M. Sugiura, H. Saito and Y. Syoyama, US pat. 1997/5602103, 1997.

10 Y. Chen, H. Zhang, X. Tian, C. Zhao, L. Cai, Y. Liu, L. Jia, H. X. Yin and C. Chen, Food Chem., 2008, 109, 484- 492.

11 J. Lafferty, A. C. Roach, R. S. Sinclair and T. G. Truscott, J. Chem. Soc., Faraday Trans. 1, 1977, 73, 416-429.

12 N. Getoff, Radiat. Phys. Chem., 1999, 55, 395-398.

13 R. Edge, A. El-Agamey, E. J. Land, S. Navaratnam and T. G. Truscott, Arch. Biochem. Biophys., 2007, 458, 104-110.

14 P. Calza, C. Minero and E. Pelizzetti, J. Chem. Soc., Faraday Trans., 1997, 93, 3765-3771.

15 J. H. Tinkler, S. M. Tavender, A. W. Parker, D. J. McGarvey, L. Mulroy and T. G. Truscott, J. Am. Chem. Soc., 1996, 118, 1756-1761.

16 H.-R. Sliwka, T. B. Melø, B. J. Foss, S. H. Abdel-Hafez, V. Partali, G. Nadolski, H. Jackson and S. F. Lockwood, Chem.-Eur. J., 2007, 13, 4458-4466.

17 B. J. Foss, G. Nadolski and S. F. Lockwood, Mini-Rev. Med. Chem., 2006, 6, 953-969.

18 S. N. Naess, A. Elgsaeter, B. J. Foss, B. Li, H. R. Sliwka, V. Partali, T. B. Melø and K. R. Naqvi, Helv. Chim. Acta, 2006, 89, $45-53$.

19 D. E. Cabelli and B. H. J. Bielski, J. Free Radicals Biol. Med., 1986, 2, 71-75.

20 M. J. Thomas and B. H. J. Bielski, J. Am. Chem. Soc., 1989, 111, 3315-3319.

21 K. R. Naqvi and T. B. Melø, Chem. Phys. Lett., 2006, 428, 83-87.

22 W. Bors, M. Saran and C. Michel, Int. J. Radiat. Biol., 1982, 41, 493-501.

23 M. McVean and D. C. Liebler, Carcinogenesis, 1997, 18, $1617-1622$.

24 E. J. Land and M. Ebert, Trans. Faraday Soc., 1967, 63, $1181-1190$. 
25 H. M. Peng, B. F. Choules, W. W. Yao, Z. Zhang, R. D. Webster and P. M. W. Gill, J. Phys. Chem. B, 2008, 112, 10367-10374.

26 A. W. Parker and R. H. Bisby, J. Chem. Soc., Faraday Trans., 1993, 89, 2873-2878.

27 M. Craw and C. Lambert, Photochem. Photobiol., 1983, 38, 241-243.

28 T. L. Miller, S. L. Willett, M. E. Moss, J. Miller and B. A. Belinka, J. Pharm. Sci., 1982, 71, 173-177.
29 F. Zsila, Z. Bikádi and M. Simonyi, Tetrahedron: Asymmetry, 2001, 12, 3125-3137.

30 K. R. Naqvi, T. H. Hassan and Y. A. Naqvi, Spectrochim. Acta, Part A, 2004, 60, 2783-2791.

31 K. R. Naqvi, T. B. Melø, H.-R. Sliwka, S. B. B. Mohamad and V. Partali, Photochem. Photobiol. Sci., 2003, 2, 381-385.

32 A. Osuka, T. Kume, G. W. Haggquist, T. Jávorfi, J. C. Lima, E. Melø and K. R. Naqvi, Chem. Phys. Lett., 1999, 313, 499-504. 